\title{
Conditional discrimination learning by pigeons: The role of training paradigms
}

\author{
DAVID R. THOMAS and HORACE GOLDBERG \\ University of Colorado, Boulder, Colorado
}

\begin{abstract}
Three groups of pigeons $(\mathrm{n}=8)$ learned a successive discrimination, 538-nm $\mathrm{S}+$ versus $576-\mathrm{nm}$ $\mathrm{S}-$, in one context, say, houselight and tone, and the reversal in another context, say, dark and noise. In Group 1, the single reversal paradigm was used; for Group 2, the problems alternated daily; and for Group 3, they alternated minute by minute. Wavelength generalization gradients obtained in both contexts revealed conditional control by context, which was weakest in Group 3.
\end{abstract}

Conditional discrimination learning refers to a situation in which the (positive or negative) value of a particular stimulus depends on the other stimulus or stimuli with which it is combined. Pigeons typically learn conditional discriminations in operant free-response situations only with great difficulty and after extensive training (Boneau \& Honig, 1964; Richards, 1979). In a study by Thomas, McKelvie, Ranney, and Moye (1981), however, such learning was achieved very rapidly. Pigeons first acquired a successive wavelength discrimination and then its reversal, each in a different physical context (e.g., houselight and tone, HL T, dark and noise, $\overline{\mathrm{HL}} \mathrm{N}$ ). The subjects were then tested for generalization in each context, and every gradient peaked at the appropriate $S+$ value, that is, that of the $\mathrm{S}+$ trained in that context.

Both the procedure and the conditional stimuli used by Thomas et al. (1981) differ from those previously used, and which of these factors accounts for the results that were obtained needs to be determined. Thomas, McKelvie, and Mah (1985) found that the auditory component of HL T played no role in the effectiveness of the compound. They also showed that striped versus plain walls were effective conditional cues, whereas background key color did not gain conditional control over a line angle discrimination in the single reversal paradigm. They suggested that pigeons may use global visual cues to identify the place in which food is obtained and may be predisposed to associate such place cues with the learning that occurred in those locations.

The present study investigates the temporal factors that may affect operant free-response conditional discrimination learning. With regard to the alternation of training conditions, the single reversal paradigm is at one end of a continuum. At the other end is the typical procedure in which the cues alternate on a minute-by-minute basis (cf. Boneau \& Honig, 1964; Richards, 1979). Intermediate between these extremes is conditional serial discrimination reversal learning (CSDR) as used by Thomas and McKelvie (1982). In their study, a wavelength discrimi-

Requests for reprints should be addressed to David R. Thomas, Department of Psychology, Campus Box 345, University of Colorado, Boulder, CO 80309. nation and its reversal were presented on alternate days, with a different line angle accompanying each problem. The birds learned the conditional relationship, and after approximately 30 days of training, they began each session by responding appropriately even before the first reinforcer had been obtained. In generalization testing, the wavelength gradients peaked at the $S+$ value appropriate to the line angle that was present.

There does not appear to be a single study that used the same set of conditional cues in the different training paradigms. In the present study, we used the conditional cues employed by Thomas et al. (1981) in order to make this comparison.

There are several reasons why the temporal sequencing of conditions may be important in conditional discrimination learning. If interference between the memories of the two problems is a factor, then the single reversal paradigm should work best because it produces the least interference, and the minute-by-minute alternation condition should be worst. However, a study by Hickis, Robles, and Thomas (1977) suggested that $\mathrm{HL} \mathrm{T} / \overline{\mathrm{HL}} \mathrm{N}$ contexts may effectively isolate memories from each other, thus eliminating any differences among the three conditions. In their experiment, on alternate days, two different interdimensional problems were employed (color positive and line angle negative; a different color negative and a different line angle positive). When the two problems were learned in the same context, generalization gradients revealed peak shifts along both dimensions, as though the birds had received intradimensional training within sessions. When the two problems were learned in different contexts, however, there were no peak shifts, and the gradients peaked at the appropriate $S+$ values. Finally, the literature on discriminative stimulus control suggests that the minute-by-minute procedure may be best. Honig, Thomas, and Guttman (1959) used two closely spaced wavelengths and contrasted the effects of massed reinforced training on $\mathrm{S}+$ followed by massed extinction on $\mathrm{S}$ - with those obtained with the normal within-session random alternation method. The former procedure yielded poor discriminative performance and no peak shifts, whereas the latter yielded excellent discriminative performance and peak shifts, suggesting the need for sub- 
jects to make repeated comparisons among the relevant stimuli for training to have its anticipated effects.

\section{METHOD}

Subjects.

The subjects were 24 experimentally naive pigeons maintained at $80 \%$ of their ad-lib weights.

Apparatus.

This apparatus was the same as that used by Thomas et al. (1981).

\section{Procedure.}

The subjects were divided into three groups $(n=8)$. On Day 1 , all birds learned to peck the response key transilluminated by a $538-\mathrm{nm}$ light, and they then earned 60 reinforcers on a variable-interval (VI) schedule with increasing mean interreinforcement intervals. On Day 2, they received further training until 40 (VI 30 -sec) reinforcers had been earned. Up to this point, the three groups were treated equally; for half of each group, HL T had been present, whereas for the other half, $\overline{\mathrm{HL}} \mathrm{N}$ had been present (Context 1). On Day 3 and for 7 succeeding days, discrimination training was given; responding was (VI 30-sec) reinforced during $\mathrm{S}+$ and extinguished in $\mathrm{S}-$. The subjects were run for $30 \mathrm{~min}$ daily. Trials were of $55-\mathrm{sec}$ duration and were separated by 5 -sec blackouts; $S+$ and $S-$ occurred in a nonsystematic order. For Groups 1 and 2, Context 1 accompanied the Day 3 training session; $S+$ was $538 \mathrm{~nm}$, and S - was $576 \mathrm{~nm}$. In Group 1, three more sessions were given under the same conditions as for Day 3, followed by four sessions of reversal training in the other context (Context 2). For Group 2, the two problems were reversed daily, such that Problem 1 (538 nm S+, $576 \mathrm{~nm} \mathrm{S-)}$ was experienced on Days 3, 5, 7, and 9 (in Context 1) and Problem $2(576 \mathrm{~nm} \mathrm{~S}+, 538 \mathrm{~nm} \mathrm{~S}-$ ) was experienced on Days $4,6,8$, and 10 (in Context 2). The change in context at the start of reversal training disrupted keypecking such that most birds in both Groups 1 and 2 had to be reshaped to keypeck in Context 2 . Several birds in Group 2 had to be reshaped again on the next day when Context 1 was reinstated. In Group 3, starting on Day 3, all four stimulus combinations were presented in a nonsystematic order, with reinforcement available in the presence of $538 \mathrm{~nm}$ in "Context 1" and $576 \mathrm{~nm}$ in "Context 2." For several subjects that did not respond in Context 2, training was interrupted, and the birds were reshaped to respond to $576 \mathrm{~nm}$, the $\mathrm{S}+$ in this context.

On the day following completion of discrimination training, all birds were tested for wavelength generalization in extinction. Keylights of $490,538,555,576$, and $606 \mathrm{~nm}$ were randomized into blocks, and eight different randomized blocks were presented to each subject. Half of each subgroup started the test in each context. The contexts were changed after every two test blocks. During generalization testing, trials were $30 \mathrm{sec}$ in duration and were separated by $5-\mathrm{sec}$ blackouts.

\section{RESULTS}

Because there were no differences in training or testing due to the counterbalancing of conditions, the data were pooled over the different subgroups. On the last sessions of Problem 1 and Problem 2 training, Group 1 yielded means of $95.2 \%$ (range $85 \%-100 \%$ ) and $95.4 \%$ (range $88 \%-100 \%$ ) of total responses to $S+$, respectively. Unlike the single reversal procedure used with Group 1, the CSDR procedure used with Group 2 permitted tracing the development of conditional control by the two contexts. By the second or third training session with each problem, the subjects began to respond appropriately to the different stimulus combinations before reinforcement was earned, and at the end of training the birds in Group 2 achieved a mean discrimination index (i.e., \% to $S+$ ) of

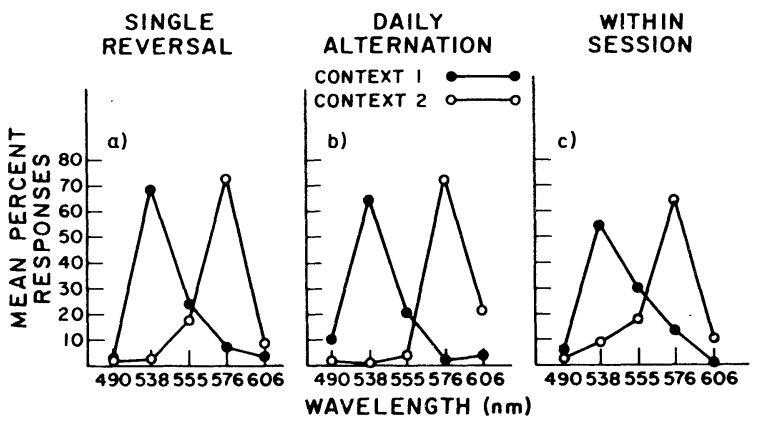

Figure 1. Mean relative generalization gradients of the three groups in each of two contexts.

$96.1 \%$ on Problem 1 (range $82 \%-100 \%$ ) and $98.2 \%$ on Problem 2 (range $90 \%-100 \%$ ). Acquisition data indicated that conditional control was slower to develop in Group 3, and on their last training session, these subjects achieved a mean discrimination index of $84.1 \%$ on Problem 1 (range $72 \%-99 \%$ ) and $84.2 \%$ on Problem 2 (range 74\%$97 \%$ ). For each subject, a pooled discrimination index was computed on the basis of the average of the two problems, and analysis of variance yielded $F(2,21)=$ $5.53, \mathrm{p}<.02$. This difference was clearly due to Group 3's performing more poorly than the other two groups. A relative generalization gradient was computed for each subject in each context; the group means of these gradients are presented in Figure 1. In both Groups 1 and 2 , HL T and HL N contexts acquired very strong conditional control, and in each of these groups, virtually every one of the individual subjects' gradients peaked at the appropriate $\mathrm{S}+$ for each context. Although considerable conditional control was also established with Group 3, it was less strong than with the other two groups. The average gradients of this group were less sharp than those of the other two groups, but the group difference becomes most apparent when individual performance is considered: Only three of the eight subjects in this group yielded gradients that peaked at the appropriate $S+$ value in both contexts.

\section{DISCUSSION}

Clearly, HL T and $\overline{\mathrm{HL}} \mathrm{N}$ contexts rapidly gain conditional control over discriminative responding in both single reversal and CSDR paradigms. The performance of Group 1 indicates that repeated comparisons between these conditional stimuli are not required during training for conditional control to develop. This has not always been the case. More extended training and the CSDR procedure have been required with other conditional cues (e.g., key color, floor tilt, etc.) in unpublished work in our laboratory. The good performance of Group 2 after so few sessions suggests that the two contexts permitted the separate encoding. storage. and retrieval of the memories of the two training experiences. thus preventing any interference between them. This is what occurred in the study by Hickis et al. (1977), in which the absence of otherwise expected peak shifts indicated the independence of odd-day and even-day memories. However, the HL $\mathrm{T} / \overline{\mathrm{HL}} \mathrm{N}$ contexts did not entirely isolate the memories of the different problems in Group 3, which suggests that it is their combination with a 24-h separation between the training conditions that produces this result. Without this temporal sepa- 
ration, some interference between conflicting memories is observed, which results in less discriminative control and less conditional control. Good discriminative control appears to be a necessary condition, but it is not a sufficient condition for the development of good conditional control.

The present study indicated that the houselight (on or off) is so salient a conditional cue that the training paradigm used makes relatively little difference. The fact that the within-session procedure degraded performance even with the potent houselight cues, however, suggests that the use of this procedure was probably responsible for the difficulty in obtaining good conditional discrimination performance in studies by Boneau and Honig (1964) and Richards (1979). The ideal training condition for the acquisition of conditional stimulus control must balance the facilitatory effects of repeated stimulus comparisons against the detrimental effects of interference between conflicting memories.

\section{REFERENCES}

Boneau, C. A. , \& HoNIG, W. K. (1964). Opposed generalization gradients based upon conditional discrimination training. Journal of $E x$ perimental Psychology, 66, 89-93.
Hickis, C. F., Robles, L., \& Thomas, D. R. (1977). Contextual stimuli and memory retrieval in pigeons. Animal Learning \& Behavior, 5 , 161-168.

Honig, W. K., Thomas, D. R., \& Guttman, N. (1959). differential effects of continuous extinction and discrimination training on the generalization gradient. Journal of Experimental Psychology, 58, 145-152.

RICHARDS, R. W. (1979). Stimulus control following training on a conditional discrimination. Animal Learning \& Behavior, 7, 309-312.

Thomas, D. R., \& McKelvie, A. R. (1982). Serial discrimination reversal learning in the pigeon: Potentiation of "pattern" by a conditional cue. Journal of Experimental Psychology: Animal Behavior Processes, 8, 274-287.

Thomas, D. R., McKelvie, A. R., \& MAH, W. (1985). The context as a conditional cue in operant discrimination reversal learning. Journal of Experimental Psychology: Animal Behavior Processes, 11, 317-330.

Thomas, D. R., McKelvie, A. R., Ranney, M., \& Moye, T. B. (1981). Interference in pigeons' long-term memory viewed as a retrieval problem. Animal Learning \& Behavior, 9, 581-586.

(Manuscript received for publication February 20, 1985.) 